\title{
Comparison of inshore zooplankton and ichthyoplankton populations of the Gulf of Maine*
}

\author{
David W. Townsend \\ Bigelow Laboratory for Ocean Sciences, McKown Point, West Boothbay Harbor, Maine 04575, USA
}

\begin{abstract}
Zooplankton and ichthyoplankton were sampled in 2 hydrographically different areas on the US Maine coast: Sullivan Harbor in eastern Maine and the Damariscotta estuary in western Maine. Sampling was conducted from late winter to early summer in each area in 1979 and 1980 . Phytoplankton chlorophyll concentrations were determined for each area in 1979. Phytoplankton and zooplankton blooms appeared to be coupled and differed in timing between areas in 1979. Timing of peak zooplankton abundances was not appreciably different between areas in 1980, but was earlier in the season than in the previous year. Times of maximum catch rates of dominant larval fish species were closely coupled to plankton dynamics.
\end{abstract}

\section{INTRODUCTION}

Seasonal cycles of zooplankton abundance and species composition in the coastal and offshore waters of the Gulf of Maine have received attention from a number of investigators (Bigelow, 1926; Clarke, 1933, 1934; Redfield, 1939, 1941; Redfield and Beal, 1940; Colton et al., 1962; Sherman, 1965, 1966, 1968, 1970; Sherman and Perkins, 1971). Their findings suggest that the cycle follows that of the phytoplankton with a peak in late spring just after the phytoplankton bloom, although at times the zooplankton reaches maximum biomass during summer (Sherman, 1965, 1966, 1968). The peak in abundance of both phytoplankton and zooplankton occurs earlier in the western Gulf of Maine and spreads gradually to the east with the onset and development of thermal stratification during spring and summer (Bigelow, 1927).

In contrast to coastal and offshore waters, zooplankton of the inshore embayments and estuaries of the Gulf of Maine are known from only a few isolated accounts and the interpretation of differences along the coast is difficult. Willey $(1913,1915)$ and Legaré and McLellan (1960) reported on the zooplankton of the Passamaquoddy Bay area; Lee (1975) and Lee and McAlice (1979a) studied the Damariscotta River estuary; and McAlice (1973) reported on the Sheepscot River-Montsweag Bay estuarine system (Fig. 1). With

- Bigelow Laboratory Contribution No. 82018 the exception of the study by McAlice (1973) the above workers sampled only at monthly or seasonal intervals and used various large mesh nets, hampering intercomparisons between areas

The ichthyoplankton of the inshore waters of the Gulf of Maine has been documented, but only for estuarine systems and nearby waters in the central area of the Maine coast (Graham and Boyar, 1965; Chenoweth, 1973; Hauser, 1973; Laroche, 1980, 1982; Shaw, 1981; Townsend and Graham, 1981). Studies in

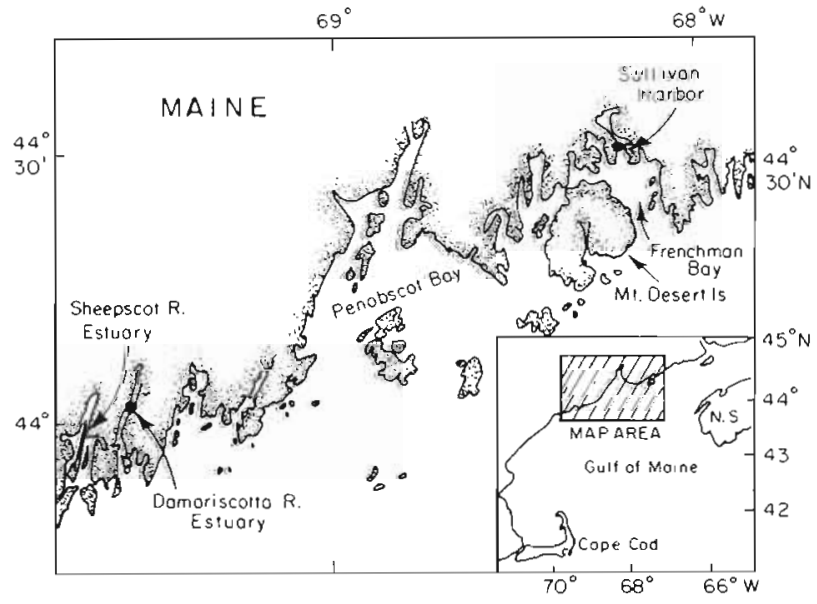

Fig. 1. Map of the 2 sampling stations in the Damariscotta River estuary and Sullivan Harbor, in relation to the US Maine coast and the Gulf of Maine. N. S.: Nova Scotia, Canada 
each of these estuaries showed that in general the highest catch rates of larval fishes occurred in the spring, which is close to the times of the spring phytoplankton and zooplankton blooms in this region.

This paper presents the results of a comparative study of the abundance and species composition of zooplankton and ichthyoplankton as they relate to hydrography and, in Year 1 of the study, phytoplankton biomass, in 2 inshore areas on the coast of Maine sampled from late winter to early summer during 1979 and 1980. The 2 sample areas, the Damariscotta estuary in western Maine and Sullivan Harbor - an embayment in eastern Maine (Fig. 1) - were chosen to represent the hydrographic variation along the Maine coast from west to east (Townsend, 1981, 1983).

\section{MATERIALS AND METHODS}

\section{Field procedures}

Weekly ichthyoplankton samples and biweekly, with some weekly, zooplankton samples were collected from January to July in 1979 and January to May in 1980 in both the Damariscotta estuary and Sullivan Harbor. Details of the study areas and sampling
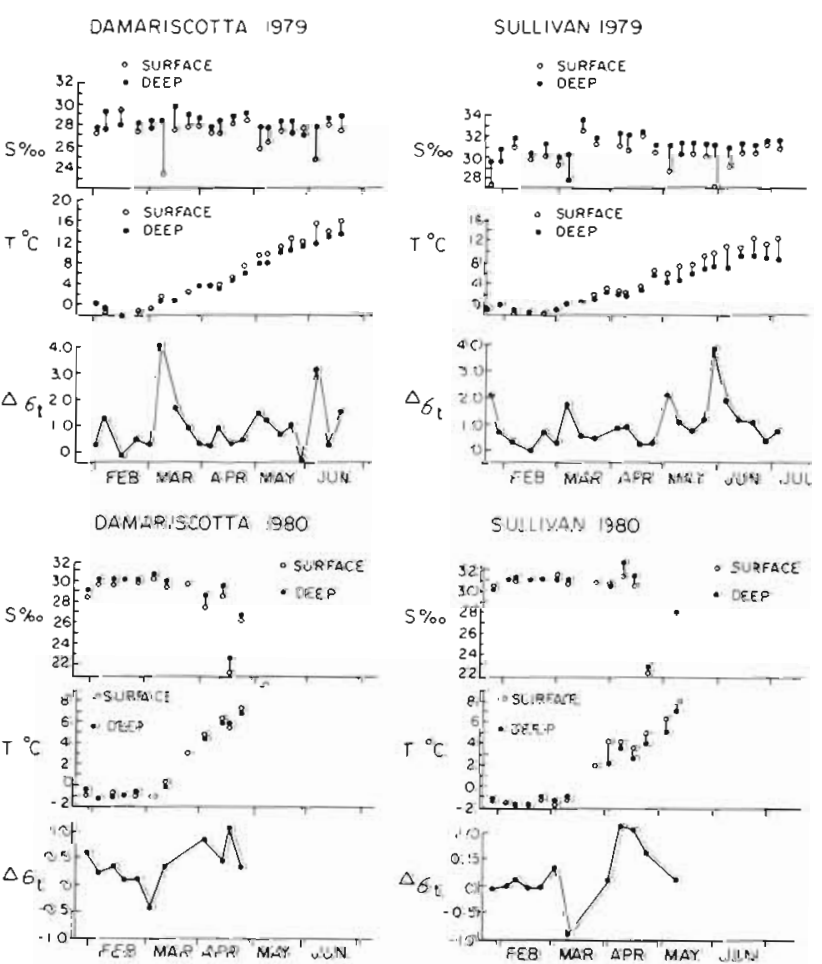

Fig. 2. Surface and deep (deep is $15 \mathrm{~m}$ in Sullivan Harbor and $25 \mathrm{~m}$ in the Damariscotta estuary) temperatures and salinities, and surface to deep differences in sigma-t for the Damariscotta estuary and Sullivan Harbor, 1979 and 1980

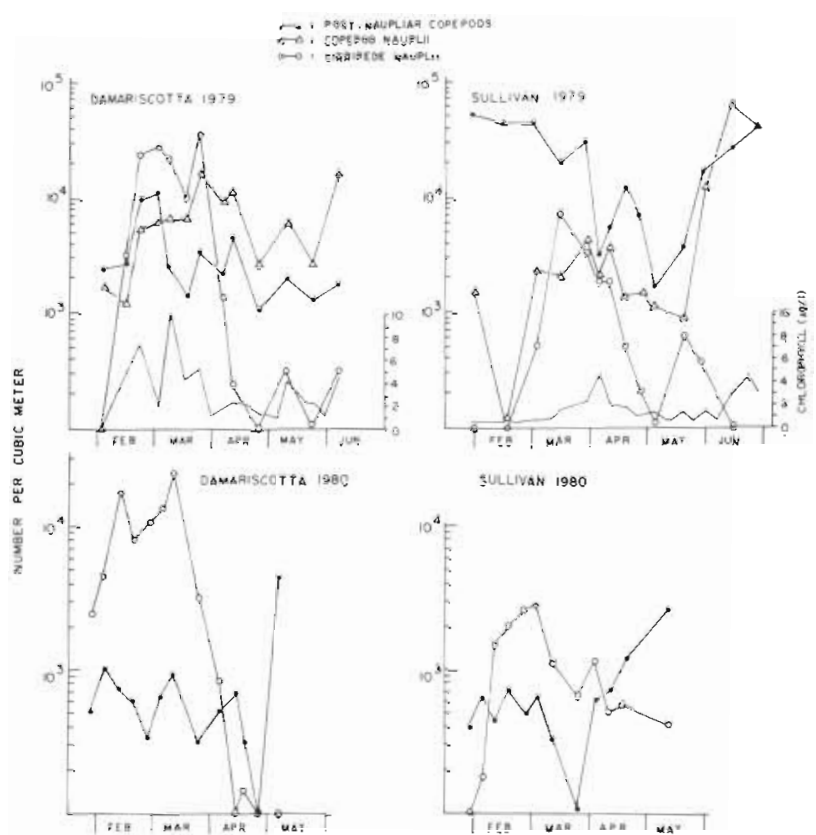

Fig. 3. Abundances of the major zooplankton groups comparable between areas for 1979 and 1980 and chlorophyll a for both areas in 1979. Mean values of either 2 or 4 chlorophyll samples are plotted. The 1979 zooplankton samples were collected with $80 \mu \mathrm{m}$ mesh nets, the 1980 samples with $165 \mu \mathrm{m}$ mesh nets

methods are given elsewhere (Townsend, 1981, 1983) and are summarized here. A $61 \mathrm{~cm}$ diameter bongo net frame was used to sample the larval fishes and a $20 \mathrm{~cm}$ diameter bongo was used on the same wire to sample the zooplankton (Posgay and Marak, 1981). The large bongo was fitted with $505 \mu \mathrm{m}$ mesh nets on each side. In $1979,80 \mu \mathrm{m}$ mesh nets were used on the small bongo initially but due to problems with net clogging a 165 $\mu \mathrm{m}$ mesh net was placed on one side of the paired bongo after the first 3 mo of sampling. Only $165 \mu \mathrm{m}$ mesh nets were used on the small bongo in 1980. A single station was sampled in each area with replicate surface and deep tows taken during midday. Temperatures and salinities were measured with a Beckman RS5-3 field salinometer-thermometer. ChIorophyll samples were taken in 1979 only.

\section{Laboratory procedures}

Zooplankton settled volumes were determined for all $20 \mathrm{~cm}$ bongo samples by allowing each to settle overnight in a graduated cylinder. Because the main sources of plankton sampling variability are generally between net tows, both between and within stations, and the least variability is caused by subsampling in the laboratory (Platt et al., 1970; Lee and McAlice, 1979b), the zooplankton species composition and 
abundance determinations were made by pooling the replicate samples. The $20 \mathrm{~cm}$ bongo samples of the same net mesh size for a particular date and area were combined and the pooled sample was diluted to 10 to 20 times the settled volume. All zooplankton in a $1 \mathrm{ml}$ aliquot were counted and identified to species, when possible, in a Sedgwick-Rafter cell. All fish larvae from one side of the preserved $61 \mathrm{~cm}$ bongo were identified and counted. The chlorophyll a determinations were made fluorometrically by the method of Yentsch and Menzel (1963) using the equations of Lorenzen (1966).

\section{RESULTS}

\section{Hydrography}

The vertical stability of the water column in each area in 1979 was apparently controlled more by salinity stratification than by temperature (Fig. 2). There were marked pulses in increased stability (top to bottom sigma-t differences) in the Damariscotta estuary in early March and early June, and several smaller pulses in Sullivan Harbor in late January, early March, early May, and a large peak in early June of that year. In

Table 1. Estimated numbers of zooplankton organisms per cubic meter, Damariscotta River estuary, 1979. Samples were collected with $80 \mu \mathrm{m}$ mesh (No. 20) plankton nets

\begin{tabular}{|c|c|c|c|c|c|c|c|c|c|c|c|c|c|}
\hline \multirow[t]{2}{*}{ Taxa } & \multicolumn{13}{|c|}{ Sample date } \\
\hline & $\begin{array}{c}1 \\
\text { Feb }\end{array}$ & $\begin{array}{c}15 \\
\text { Feb }\end{array}$ & $\begin{array}{c}23 \\
\text { Feb }\end{array}$ & $\stackrel{2}{\mathrm{MaJ}}$ & $\begin{array}{c}8 \\
\text { MaI }\end{array}$ & $\begin{array}{c}16 \\
\mathrm{Mar}\end{array}$ & $\begin{array}{c}22 \\
\text { MaI }\end{array}$ & $\begin{array}{c}6 \\
\mathrm{Apr}\end{array}$ & $\begin{array}{c}11 \\
\text { Apr }\end{array}$ & $\begin{array}{c}25 \\
\text { Apr }\end{array}$ & $\begin{array}{c}9 \\
\text { May }\end{array}$ & $\begin{array}{c}23 \\
\text { May }\end{array}$ & $\begin{array}{c}6 \\
\text { Jun }\end{array}$ \\
\hline \multicolumn{14}{|l|}{ Phylum Protozoa } \\
\hline \multicolumn{14}{|l|}{ Class Rhizopoda } \\
\hline Unidentified foramumferans & 67 & & & 208 & & 49 & 235 & 31 & & & & & \\
\hline \multicolumn{14}{|l|}{ Class Ciliata } \\
\hline Tintinnopsis sp. & & & & 5208 & 12522 & 12097 & 62832 & 3047 & 1700 & & & & \\
\hline Unidentified tintinnids & & & & 208 & & & & & & & & & \\
\hline \multicolumn{14}{|l|}{ Phylum Aschelminthes } \\
\hline \multicolumn{14}{|l|}{ Class Nematoda } \\
\hline Unident 1 fied nematodes & 67 & 45 & & 416 & 180 & 49 & & & 34 & & & 70 & 2124 \\
\hline \multicolumn{14}{|l|}{ Class Rotifera } \\
\hline Synchaeta sp. & 89 & 23 & & 416 & 361 & 12487 & 33529 & 1269 & 1350 & 1953 & 3868 & 94 & 432 \\
\hline Trichocerca sp. & & & & & & 49 & & 31 & & & & & \\
\hline Keratella sp. & & & & & & 117 & & & 33 & 31 & 33 & & \\
\hline \multicolumn{14}{|l|}{ Phylum Mollusca } \\
\hline \multicolumn{14}{|l|}{ Class Gastropoda } \\
\hline Littonna sp. eggs & & & & & & & & & 17 & & & & \\
\hline Unudentufued gastropod larvae & 45 & 23 & & 208 & & & & 31 & 67 & & 66 & 23 & 72 \\
\hline \multicolumn{14}{|l|}{ Class Bivalvia } \\
\hline Unidentfied stranght-hinge larvae & 22 & & & & & & & & & & & 47 & 4968 \\
\hline \multicolumn{14}{|l|}{ Phylum Annelida } \\
\hline \multicolumn{14}{|l|}{ Class Polychaeta } \\
\hline Unidentified trochophore larvae & & & & 625 & 630 & 49 & 235 & 285 & 1650 & 6449 & 4229 & 2894 & 468 \\
\hline Unidentified seteger larvae & & & & 208 & & & & & 17 & 31 & 196 & 1458 & 432 \\
\hline Phylum Arthropoda & & & & & & & & & & & & & \\
\hline Class Crustacea & & & & & & & & & & & & & \\
\hline Subclass Cirripedia & & & & & & & & & & & & & \\
\hline Cirripede nauplı & 89 & 3307 & 24342 & 27500 & 22972 & 10292 & 39529 & 1492 & 250 & 62 & 327 & 117 & 324 \\
\hline Cirnipede cypnds & & & & & & & & 984 & 233 & & & & \\
\hline Subclass Copepoda & & & & & & & & & & & & & \\
\hline Acartia longiremis (Lilljeborg) adults & 291 & 638 & 800 & 869 & 360 & 341 & 235 & 158 & 2000 & 62 & 295 & 367 & 432 \\
\hline Acartia sp. copepodites & 45 & 91 & & & & 146 & 470 & 857 & 966 & 620 & 786 & 305 & 1152 \\
\hline Acartra sp. naupl.i & 985 & 729 & 2971 & 4791 & 3243 & 3853 & 12588 & 6000 & 7250 & 1426 & 5081 & 1647 & 7668 \\
\hline Calanus finmarchicus (Gunnerus) adults & 22 & & & & & & & & & & & & \\
\hline Centropages hamarus (Lilljeborg) naupliz & & & & & & & & & 516 & 124 & 229 & 23 & 288 \\
\hline Eurytemora herdmani Thompson and Scott adults & 45 & & & & & & & & 17 & & & 47 & 36 \\
\hline E. herdmani copepodites & & & & & & & & & 216 & 62 & 327 & 47 & \\
\hline E. herdmani nauplii & & & & & & 975 & 352 & 1777 & 783 & 775 & 360 & 141 & 216 \\
\hline Microsetella norvegrca (Boeck) adults and copepodites & 1432 & 1573 & 8800 & 10000 & 1982 & 878 & 2235 & 253 & 933 & 341 & 163 & & \\
\hline M. norvegica naupliı & 22 & & & & & 98 & 117 & 190 & 200 & & 32 & 47 & 288 \\
\hline Oithona similss Claus adults and copepodites & 22 & 182 & 457 & 208 & & 49 & 117 & 31 & 50 & & 33 & & \\
\hline O. similss nauplii & 156 & 45 & 114 & & 270 & & 117 & & 67 & & & 70 & \\
\hline Pseudocalanus minutus (Krover) adults & 470 & 23 & 114 & 208 & 90 & & 117 & 31 & & & 66 & 94 & \\
\hline P. minutus copepodites & 89 & 68 & 114 & & 90 & 146 & 470 & 380 & 133 & 15 & 33 & 117 & 72 \\
\hline P. minurus nauplii & 380 & 205 & 114 & 208 & & 195 & 235 & 63 & 150 & 46 & 33 & 23 & \\
\hline Temora Longicornis (Muller) adults & 22 & 136 & & 208 & 90 & & & 94 & 67 & & 98 & 70 & 36 \\
\hline T. longicornis copepodites & & & & & & & & 539 & 400 & 62 & 327 & & 180 \\
\hline T. Jongscornis nauplii & 134 & 228 & 1828 & 1250 & 2702 & 1414 & 3647 & 1333 & 1083 & 434 & 426 & 494 & 360 \\
\hline Unid. Harpacticold adults and copepodites & 156 & 23 & & & 90 & & & & 34 & 31 & 33 & 23 & 72 \\
\hline Unid. Harpacticoid nauplii & & & & & & 149 & 117 & & 34 & 31 & 66 & 329 & 1836 \\
\hline Unid. copepod nauplij & 45 & & & & 361 & 243 & 352 & 127 & 133 & 31 & & & 72 \\
\hline Unid. copepod eggs & 67 & 68 & 114 & & & 146 & 588 & 31 & 366 & 124 & 33 & & \\
\hline Phylum Chordata & & & & & & & & & & & & & \\
\hline Class Larvacea & & & & & & & & & & & & & \\
\hline Oikopleura sp. & & & & & & & & & & & & 47 & \\
\hline Other - & & & & & & & & & & & & & \\
\hline Unid. invertebrate eggs & 268 & 114 & 800 & 1250 & 270 & 946 & 235 & 158 & 167 & 32 & 262 & & 936 \\
\hline Hansen's nauplii & & & & 208 & & & & & & & & & \\
\hline
\end{tabular}


each instance, the peaks in stability were the result of influxes of low salinity surface water. These events were synchronous between areas and most likely represented maxima in freshwater runoff from land into the systems. The average salinities in 1979 were persistently lower in the Damariscotta than in Sullivan. The water temperatures were similar between areas until early March when warming proceeded more rapidly in the Damariscotta; the difference in water temperature was about 1 to $1.5^{\circ} \mathrm{C}$ in March.

In 1980 , there was very little vertical stability in either area until March (Fig. 2). It was late March when the water column began to stabilize in the Damariscotta estuary and early April in Sullivan. The differences between surface and deep salinities were very slight in each area, with no significant fresh water additions until late April. However, these salinity differences were still more important than temperature in stabilizing the water column. The water temperatures in the Damariscotta estuary were persistently higher than those in Sullivan Harbor throughout the sampling period.

Table 2. Estimated numbers of zooplankton organisms per $\mathrm{m}^{3}$, Sullivan Harbor, 1979. Samples were collected with $80 \mu \mathrm{m}$ mesh (No. 20) plankton nets

\begin{tabular}{|c|c|c|c|c|c|c|c|c|c|c|c|c|c|c|}
\hline \multirow[t]{2}{*}{ Taxa } & \multicolumn{14}{|c|}{ Sample date } \\
\hline & $\begin{array}{c}31 \\
\text { Jan }\end{array}$ & $\begin{array}{c}16 \\
\mathrm{Feb}\end{array}$ & $\stackrel{1}{\mathrm{Mar}}$ & $\begin{array}{c}15 \\
\text { Mar }\end{array}$ & $\begin{array}{c}29 \\
\text { Mar }\end{array}$ & $\begin{array}{c}5 \\
\text { Apr }\end{array}$ & $\begin{array}{c}10 \\
\text { Apr }\end{array}$ & $\begin{array}{c}19 \\
\text { Apr }\end{array}$ & $\begin{array}{c}26 \\
\text { Apr }\end{array}$ & $\begin{array}{c}3 \\
\text { May }\end{array}$ & $\begin{array}{c}17 \\
\text { May }\end{array}$ & $\begin{array}{c}31 \\
\text { May }\end{array}$ & $\begin{array}{c}14 \\
\text { Jun }\end{array}$ & $\begin{array}{c}28 \\
\text { Jun }\end{array}$ \\
\hline \multicolumn{15}{|l|}{ Phylum Protozoa } \\
\hline \multicolumn{15}{|l|}{ Class Rhizopoda } \\
\hline Unidentified foraminuferans & 26 & & & & & & & & & & & & & \\
\hline \multicolumn{15}{|l|}{ Class Ciliata } \\
\hline Unıdentified tintinuds & & & & 72 & & & & 28 & & & 2113 & & & 3384 \\
\hline \multicolumn{15}{|l|}{ Phylum Aschelminthes } \\
\hline \multicolumn{15}{|l|}{ Class Nematoda } \\
\hline Unidentified nematodes & & & 120 & & & & & & 215 & 274 & & & 2526 & \\
\hline \multicolumn{15}{|l|}{ Class Rotifera } \\
\hline Synchaetasp. & 26 & & & 72 & 436 & 156 & 345 & 169 & 86 & 55 & 905 & 952 & 2526 & 12615 \\
\hline Trichocerca sp. & & & & & & & & & & & & & 421 & \\
\hline \multicolumn{15}{|l|}{ Phylum Mollusca } \\
\hline \multicolumn{15}{|l|}{ Class Gastropoda } \\
\hline Lutrorina sp eggs & & & & & & 156 & 86 & 56 & 258 & 274 & 302 & & 210 & 307 \\
\hline Unidentified gastropod larvae & & 24 & 80 & 214 & 436 & & & & 215 & 55 & 603 & 666 & 421 & \\
\hline \multicolumn{15}{|l|}{ Class Bivalvna } \\
\hline Urudentified straight-hinge larvae & & & & & & & & & 86 & 55 & & 4571 & 18315 & 7692 \\
\hline \multicolumn{15}{|l|}{ Phylum Annelida } \\
\hline \multicolumn{15}{|l|}{ Class Polychaeta } \\
\hline Unidentified trochophore larvae & & & 40 & 286 & 727 & & 259 & 84 & 129 & 164 & 302 & 381 & 1052 & 923 \\
\hline Undentified seteger larvae & & & & & & 312 & 345 & 56 & 86 & & 302 & 571 & 1894 & 923 \\
\hline Phylum Arthropoda & & & & & & & & & & & & & & \\
\hline Class Crustacea & & & & & & & & & & & & & & \\
\hline Subclass Branchiopoda & & & & & & & & & & & & & & \\
\hline Evadne nordmani Loven & & & & & & & & & & & & 95 & 1263 & 1538 \\
\hline Subclass Cirripedıa & & & & & & & & & & & & & & \\
\hline Cirripede nauplı & 52 & 24 & 520 & 3450 & 3345 & 1875 & 1898 & 507 & 215 & 109 & 603 & 381 & & \\
\hline Cirripede cyprids & & & & & & & & & & & & & 210 & \\
\hline Subclass Copepoda & & & & & & & & & & & & & & \\
\hline Acartia longiremis (Lilljeborg) adults & & 24 & 80 & 72 & & & 86 & & & 164 & & 666 & 1052 & \\
\hline Acartia sp. copepodites & & & & & & & & 56 & 129 & 109 & & 95 & 421 & 923 \\
\hline Acartia sp. nauplin & 234 & & 320 & 931 & 1309 & 625 & $112 \mathrm{~J}$ & 591 & 387 & 383 & 1509 & 1142 & 3368 & 1538 \\
\hline Calanus finmarchicus (Gunnerus) nauphi & 104 & 24 & & & & & & 28 & & & & 190 & & \\
\hline Eurytemora herdmani Thompson and Scott nauphii & & & & & & & 259 & 84 & & 55 & & & & \\
\hline Microsetella norvegica (Boeck) adults and copepodites & 51588 & 45425 & 45360 & 20274 & 32581 & 3281 & 4831 & 8535 & 4258 & 657 & 3018 & 11142 & 20842 & 35692 \\
\hline M. norvegica nauplu & 26 & & 40 & & & & 86 & 56 & 559 & 109 & 905 & 9238 & 57052 & 30153 \\
\hline$M$. norvegica eggs & 156 & 121 & 120 & 143 & 145 & & 259 & 3690 & 2236 & 218 & 905 & 5238 & 4421 & 4923 \\
\hline Oithona sumilis Claus adults and copepodites & 807 & 340 & 360 & 358 & & & 86 & 140 & 86 & & & 95 & & 923 \\
\hline O. similis naupliı & 859 & & 1240 & 788 & 1891 & 781 & 1380 & 338 & 344 & 438 & & 1047 & 1894 & 4307 \\
\hline Pseudocalanus minutus (Kroyer) adults & 52 & 73 & & 72 & & & & 84 & 43 & 55 & & & & \\
\hline P. minutus copepodites & & 48 & 40 & 72 & & & & & & 219 & & 285 & 631 & 615 \\
\hline P. minutus nauplt & 234 & 97 & 320 & & & & & 84 & 43 & 55 & & 95 & 842 & 1538 \\
\hline Temora longicornis (Muller) adults & & & & & & & & & & 55 & & & 842 & \\
\hline T. longicornis copepodites & & 24 & & & & & & 140 & & 55 & & & & 307 \\
\hline$T$ longicomus nauplii & 234 & 24 & 520 & 358 & 827 & 625 & 776 & 309 & 129 & 109 & & 1238 & 1473 & 3384 \\
\hline Und. Harpacticoid adults and copepodites & & & & 72 & & & 690 & 56 & 473 & 218 & & 95 & 631 & 307 \\
\hline Unid. Harpacticoid nauplii & & & & 72 & & & 172 & & 43 & & & & & \\
\hline Und Copepod nauplil & & & & & & & & & 43 & & & & & \\
\hline Subclass Decapoda & & & & & & & & & & & & & & \\
\hline Hyas sp zoea & & & & & & & & 28 & & & & & & \\
\hline Phylum Chordata & & & & & & & & & & & & & & \\
\hline Class Larvacea & & & & & & & & & & & & & & \\
\hline Fritillana borealis Lohmann & & & & & & & & & 43 & & 603 & 952 & 210 & \\
\hline Other - & & & & & & & & & & & & & & \\
\hline Und. invertebrate eggs & & & & & 3927 & 781 & 345 & & 903 & 109 & 3924 & 1047 & 421 & \\
\hline
\end{tabular}




\section{Chlorophyll}

Chlorophyll a concentrations in 1979 reached peaks in the Damariscotta estuary in late February and early to mid-March followed by lower values and a third peak in May (Fig. 3). There were 2 peaks in chlorophyll in Sullivan Harbor, in early April and June. The chlorophyll levels in Sullivan Harbor were generally lower than the Damariscotta.

\section{Zooplankton}

The estimated abundances and species composition of zooplankton are summarized in Tables 1 to 4 . The tintinnid, Tintinnopsis sp. had the highest peak abundance in the Damariscotta estuary in 1979, reaching its peak abundance on 22 March, as did the other 2 abundant taxa, the rotifer Synchaeta sp. and cirripede nauplii. The species composition in Sullivan Harbor

Table 3. Estimated numbers of zooplankton organisms per $\mathrm{m}^{3}$. Damariscotta River estuary, 1980. Samples were collected with 165 $\mu \mathrm{m}$ mesh plankton nets

\begin{tabular}{|c|c|c|c|c|c|c|c|c|c|c|c|c|c|}
\hline \multirow[t]{2}{*}{ Taxa } & \multicolumn{13}{|c|}{ Sample date } \\
\hline & $\begin{array}{l}29 \\
\mathrm{Jan}\end{array}$ & $\stackrel{4}{\text { Feb }}$ & $\begin{array}{c}12 \\
\text { Feb }\end{array}$ & $\begin{array}{c}19 \\
\text { Feb }\end{array}$ & $\begin{array}{c}26 \\
\text { Feb }\end{array}$ & $\stackrel{4}{\text { Mar }}$ & $\begin{array}{c}11 \\
\text { Mar }\end{array}$ & $\begin{array}{c}25 \\
\text { Mar }\end{array}$ & $\begin{array}{c}4 \\
\text { Apr }\end{array}$ & $\begin{array}{c}14 \\
\text { Apr }\end{array}$ & $\begin{array}{c}18 \\
\mathrm{Apr}\end{array}$ & $\begin{array}{c}25 \\
\text { Apr }\end{array}$ & $\begin{array}{c}6 \\
\text { May }\end{array}$ \\
\hline \multicolumn{14}{|l|}{ Phylum Protozoa } \\
\hline \multicolumn{14}{|l|}{ Class Rhızopoda } \\
\hline Unidentified foraminiferans & & & & & & 27 & & & & & 6 & & 20 \\
\hline \multicolumn{14}{|l|}{ Class Cillata } \\
\hline Parafavella sp & & & & & & & & & 21 & & & & \\
\hline Und tuntinnids & & & & & & & & & & 7 & 6 & & \\
\hline \multicolumn{14}{|l|}{ Phylum Aschelminthes } \\
\hline \multicolumn{14}{|l|}{ Class Nematoda } \\
\hline Unid nematodes & & & & & & & & & & & 30 & & \\
\hline \multicolumn{14}{|l|}{ Class Ronfera } \\
\hline Synchaera sp. & & & & 14 & & & 3.3 & & 7 & & & 15 & 62 \\
\hline \multicolumn{14}{|l|}{ Phylum Mollusca } \\
\hline \multicolumn{14}{|l|}{ Class Gastropoda } \\
\hline Littorina sp. eggs & & & & & 30 & & & & 102 & 66 & 105 & 5 & 41 \\
\hline Unid gastropod larvae & & 16 & 38 & & & & & & 7 & & & & 62 \\
\hline \multicolumn{14}{|l|}{ Phylum Annelida } \\
\hline \multicolumn{14}{|l|}{ Class Polychaeta } \\
\hline \multicolumn{14}{|l|}{ Unid. trochophore larvae } \\
\hline Unid seteger larvae & & & & & 15 & & & & 21 & & 6 & & 167 \\
\hline \multicolumn{14}{|l|}{ Phylum Arthropoda } \\
\hline Class Crustaced & & & & & & & & & & & & & \\
\hline Subclass Cirrepedia & & & & & & & & & & & & & \\
\hline Cirnpede nauplii & 2506 & 4789 & 17574 & 8344 & 11363 & 1.3713 & 24117 & 3285 & 856 & 22 & 154 & & \\
\hline Cimipede cyprids & & & & & & & 33 & 1916 & 600 & 37 & 49 & & \\
\hline Subclass Copepoda & & & & & & & & & & & & & \\
\hline Acartia claust Giesbrechl adults & & & & & & & & & & & & & 20 \\
\hline Acartiasp longiremss (Lit)jeborg) adults & 223 & 356 & 321 & 229 & 61 & 138 & 131 & 45 & 23 & 102 & 43 & & 833 \\
\hline Acartia sp copepodites & 140 & 348 & 302 & 158 & 122 & 385 & 262 & 274 & 358 & 249 & 136 & & 2187 \\
\hline Acartia sp. naupli & & 8 & 38 & 43 & & 55 & 65 & 23 & 7 & 29 & 12 & & 1145 \\
\hline Calanus finmarchncu |Gunnerusl adults & & & & 14 & & 55 & & & & & & & \\
\hline C finmarchicus nauplil & & & & & & & & 45 & & & 6 & & \\
\hline Centropages hamatus (Lilljebolg) adults & & & & & & & & & & & & & 62 \\
\hline C. hamatus nauplı & & & & & & & & & & & & & 62 \\
\hline Eurytemora herdman! Thompson and Scotl adults & 25 & 25 & & & 46 & & 65 & & 7 & 22 & 37 & & 146 \\
\hline E. herdmani copepodites & & & 19 & & & & & & & & & & 333 \\
\hline E herdmani nauplì & & & & & & 55 & 65 & & & & & & 83 \\
\hline Oithona similis Claus adults and copepodites & 16 & 124 & & & 46 & & 164 & & 29 & 22 & & & 20 \\
\hline Paratholistris crom (Kroyer) adults and copepodites & & & & 28 & & & & & & & & & \\
\hline Pseudocalanus minutus (Kroyer) adults & 58 & 49 & 19 & & 1.5 & 55 & 164 & & & & & & 41 \\
\hline$P$ minutus copepodites & 24 & 17 & 19 & & 15 & 27 & 98 & & & 7 & & & \\
\hline P. minutus nauplin & & 8 & 38 & & 30 & & 131 & 91 & 29 & 7 & 18 & & \\
\hline Temora longicornis (Muller) adults & 66 & 66 & 75 & 72 & 15 & & 6.5 & & 7 & 22 & 12 & & 167 \\
\hline T. longicomis copepodites & & & & & & & & & 88 & 161 & 49 & & 875 \\
\hline$T$. longicornis naupliı & & & & 14 & & & & 46 & 7 & & 6 & & 312 \\
\hline Tortanus discaudaths (Thompson and Scott) adults & & 17 & & 14 & & 27 & & & 7 & & & & \\
\hline Unid. Harpacticoid adults and copepodites & & & 19 & 57 & & & & & & 117 & 42 & 5 & 41 \\
\hline Unid. Harpacticold nauplı! & & & & 43 & 30 & & & & & & 18 & 10 & 20 \\
\hline Unid. copepod nauplii & & 8 & & & & & & & & & & & 41 \\
\hline Subclass Decapoda & & & & & & & & & & & & & \\
\hline Hyas sp. zoea' & & & & & & & & & & 14 & & 5 & \\
\hline Phylum Chaelognatha & & & & & & & & & & & & & \\
\hline Sagutta elegans Verrill & 8 & & & & & & & & & & & & \\
\hline Other - & & & & & & & & & & & & & \\
\hline Hansen's nauplii & & & & & & & & & 14 & & 6 & & \\
\hline Unid. invertebrate eggs & 8 & & & 14 & & & & & & & & & \\
\hline
\end{tabular}


in 1979 was different from the Damariscotta (Tables 1 and 2). The dominant species in Sullivan was the harpacticoid copepod Microsetella norvegica which was at peak abundance on the first sample date, 31 January.

Comparisons of the 1979 and 1980 zooplankton results are complicated by the fact that most of the smaller organisms were undersampled by the $165 \mu \mathrm{m}$ nets used in 1980. A comparison of the $165 \mu \mathrm{m}$ and 80 $\mu \mathrm{m}$ mesh nets is given in Table 5 . In particular, Tintinnopsis sp., Synchaeta sp., polychaete trochophore larvae, adult and copepodid $M$. norvegica, and all copepod nauplii were undersampled in 1980, whereas the adult and copepodid stages of most copepods and cirripede nauplii were sampled more representatively. The dominant zooplankters in each area in 1980 were cirripede nauplii (Tables 3 and 4) which reached peak abundances earlier than the previous year.

Table 4. Estimated numbers of zooplankton organisms per $\mathrm{m}^{3}$. Sullivan Harbor, 1980. Samples were collected with $165 \mu \mathrm{m}$ mesh plankton nets

\begin{tabular}{|c|c|c|c|c|c|c|c|c|c|c|c|c|}
\hline \multirow[t]{2}{*}{ Taxa } & \multicolumn{12}{|c|}{ Sample date } \\
\hline & $\begin{array}{c}28 \\
\mathrm{Jan}\end{array}$ & $\begin{array}{c}5 \\
\text { Feb }\end{array}$ & $\begin{array}{c}11 \\
\text { Feb }\end{array}$ & $\begin{array}{c}18 \\
\text { Feb }\end{array}$ & $\begin{array}{l}25 \\
\text { Feb }\end{array}$ & $\begin{array}{c}3 \\
\text { Mar }\end{array}$ & $\begin{array}{c}10 \\
\text { Mar }\end{array}$ & $\begin{array}{c}26 \\
\text { Mar }\end{array}$ & $\begin{array}{c}3 \\
\text { Apr }\end{array}$ & $\begin{array}{c}10 \\
\text { Apr }\end{array}$ & $\begin{array}{c}17 \\
\text { Apr }\end{array}$ & $\begin{array}{c}11 \\
\text { May }\end{array}$ \\
\hline \multicolumn{13}{|l|}{ Phylum Protozoa } \\
\hline \multicolumn{13}{|l|}{ Class Ciliata } \\
\hline Unid. Untunneds & 8 & & & & & & & & & & & \\
\hline \multicolumn{13}{|l|}{ Class Rhizopoda } \\
\hline Unid. foramuniferans & 8 & & & 110 & 48 & 28 & 8 & 25 & 17 & 17 & 48 & 16 \\
\hline \multicolumn{13}{|l|}{ Phylum Aschelminthes } \\
\hline \multicolumn{13}{|l|}{ Class Nematoda } \\
\hline Unid. nematodes & 8 & & 10 & 24 & 8 & 9 & 23 & 8 & & 17 & & 32 \\
\hline \multicolumn{13}{|l|}{ Class Rotifera } \\
\hline Synchaetasp. & 8 & & & 86 & & & 8 & & & 9 & & 275 \\
\hline \multicolumn{13}{|l|}{ Phylum Mollusca } \\
\hline \multicolumn{13}{|l|}{ Class Gastropoda } \\
\hline Litronna sp. eggs & & & & & & & & & & 388 & 95 & 210 \\
\hline Unid. gastropod larvae & 16 & & 20 & 37 & 56 & 104 & 31 & 8 & & 9 & & 97 \\
\hline \multicolumn{13}{|l|}{ Class Bivalvia } \\
\hline Unid. straight-hinge larvae & & & & & 8 & 8 & & & & 35 & & 161 \\
\hline \multicolumn{13}{|l|}{ Phylum Annelida } \\
\hline \multicolumn{13}{|l|}{ Class Polychaeta } \\
\hline Unid. trochophore larvae & & & & & & 19 & 31 & 8 & 26 & 17 & 35 & \\
\hline Unid. seteger larvae & & & & & & 19 & & & 8 & & & \\
\hline Phylum Arthropoda & & & & & & & & & & & & \\
\hline Class Crustacea & & & & & & & & & & & & \\
\hline Subclass Branchiopoda & & & & & & & & & & & & \\
\hline Evadne nordmani Loven & & & & & & & & & & & & 32 \\
\hline Subclass Cirrspedia & & & & & & & & & & & & \\
\hline Cirripede nauplii & & 187 & 1500 & 2006 & 2862 & 2880 & 1138 & 666 & 1164 & 502 & 584 & 420 \\
\hline Cirripede cyprids & & & & & & 9 & & 77 & 191 & 405 & 48 & 16 \\
\hline Subclass Copepoda & & & & & & & & & & & & \\
\hline Acartia clausi Giesbrecht adults & & & & & & & & & & & 12 & \\
\hline Acartia longiremis (Lilljeborg) adults & 66 & 8 & 30 & 12 & & & 31 & 8 & 147 & 141 & 346 & 1116 \\
\hline Acartia sp. copepodites & 8 & 24 & 20 & & & 9 & 17 & 25 & 226 & 326 & 358 & 647 \\
\hline Acartia sp. naupli & & & & & 8 & 19 & & 8 & 34 & 26 & 83 & 178 \\
\hline Calanus finmarchicus (Gunnerus) adults & 8 & & & 12 & & & & & & & 24 & \\
\hline Eurytemora herdmani Thompson and Scott adults & 16 & 8 & & & & & & 8 & & 35 & & \\
\hline E. herdmani copepodites & & & & & & & & 8 & & 26 & 12 & \\
\hline E. herdmani naupliı & & & & & & & & & 8 & & & \\
\hline Microsetella norvegica (Boeck) adults and copepodites & 8 & 33 & 30 & 98 & 40 & 38 & & & & 88 & 36 & 81 \\
\hline Oithond similis Claus adults and copepodites & 223 & 464 & 230 & 344 & 233 & 390 & 147 & & 113 & 44 & 24 & 194 \\
\hline O. simulis naupli: & & & & 37 & & & 8 & & & 9 & & \\
\hline Pseudocalanus minutus (Kroyer) adults & 33 & 81 & 70 & 123 & 104 & 85 & 46 & & 26 & 9 & 48 & 291 \\
\hline P. minutus copepodites & 8 & 16 & 40 & 110 & 112 & 152 & 70 & 42 & 43 & 44 & 203 & 194 \\
\hline$P$. minutus nauplsi & 8 & 57 & & 172 & 88 & 152 & 70 & 42 & 43 & 26 & 36 & \\
\hline Temora longicornis (Muller) adults & & & & & & & & & 17 & 35 & 60 & 178 \\
\hline T. longrcorris copepodites & & & 10 & 12 & & & & & 26 & 9 & 108 & 81 \\
\hline T. Iongicomis nauplii & & & & & & 9 & & & & & 24 & \\
\hline Tortanus discaudatus (Thompson and Scott) adults & 8 & & & & & & & & & & & 48 \\
\hline T. discaudatus copepodites & 8 & & & & & & & & & & & \\
\hline Unid. Harpacticold adults and copepodites & 16 & 8 & & 24 & 16 & & 23 & 17 & & 26 & 24 & 16 \\
\hline Unid. Harpacticold nauplii & & & & & & & & 17 & & & & \\
\hline Unid. copepod adults & 8 & 16 & 10 & & & & & & & & & \\
\hline Unid. copepod eggs & & & & & & & & & & 26 & & \\
\hline Phylum Echunodermata & & & & & & & & & & & & \\
\hline Unid. pluteus larvae & & & & & & & & & & & & 97 \\
\hline Phylum Chordata & & & & & & & & & & & & \\
\hline Class Larvacea & & & & & & & & & & & & \\
\hline Fritilland borealis Lohmann & & & & & & & & & 8 & 9 & & 954 \\
\hline Other - & & & & & & & & & & & & \\
\hline Undd. invertebrate eggs & & & & 184 & 24 & & 8 & 8 & 8 & & & \\
\hline
\end{tabular}




\section{Ichthyoplankton}

A total of 24 species of larval fishes were caught during this study. The catch rates and seasonalities for all species caught in both sample areas are given in Tables 6 to 9. The dominant species for both the Damariscotta estuary and Sullivan Harbor was Pholis gunnellus. Other dominant species occurring in each area included 3 cottid congeners, Myoxocephalus scorpius, $M$. aenaeus and $M$. octodecempspinosus, and the stichaeid Lumpenus lumpretaeformis. These results, for the Damariscotta, are similar to those reported previously for this area (Graham and Boyar, 1965; Chenoweth, 1973; Hauser, 1973; Laroche, 1980, 1982;
Shaw, 1981). A complete discussion of the ecology of the cottid larvae is given by Laroche (1982).

Although differing somewhat in relative abundances, the late winter larval fish faunas in Sullivan Harbor and the Damariscotta estuary were quite similar. However, they differed considerably in species composition later in the season. In particular, Liparis atlanticus and Ammodytessp. larvae were abundant in Sullivan Harbor, but were only poorly represented in the Damariscotta. Conversely, Osmerus mordax and fall-spawned Clupea harengus larvae were abundant in the Damariscotta and rare in Sullivan Harbor. There are no previously published accounts of the fish larvae from Sullivan Harbor or waters nearby with which to

Table 5. Comparison of counts and abundance estimates of zooplankton sampled in the Damariscotta River estuary, 11 April 1979 , with a $20 \mathrm{~cm}$ Bongo net equipped with an $80 \mu \mathrm{m}$ mesh net on the port side and a $165 \mu \mathrm{m}$ mesh net on the starboard side. Two surface and deep $10 \mathrm{~min}$ tows were taken and the samples pooled. The No. 20 net sample was diluted to $2000 \mathrm{ml}$ and the No. 10 net sample was diluted to $1000 \mathrm{ml}$. A $1 \mathrm{ml}$ aliquot from each was counted. Organisms were divided into rough size categories

\begin{tabular}{|c|c|c|c|c|}
\hline \multirow{2}{*}{ Taxon } & \multicolumn{2}{|c|}{ No. $20 \mathrm{Net}$} & \multicolumn{2}{|c|}{ No. $10 \mathrm{Net}$} \\
\hline & Raw count & No. $\mathrm{m}^{-3}$ & Raw count & No. $\mathrm{m}^{-3}$ \\
\hline \multicolumn{5}{|l|}{ Smaller organisms } \\
\hline Keratella sp. & 8 & 133 & - & - \\
\hline Tintinnopsis sp. & 102 & 1700 & 2 & 14 \\
\hline Synchaeta sp. & 81 & 1350 & 1 & 7 \\
\hline Unid. gastropod larvae & 4 & 67 & - & - \\
\hline Unid. polychaete trochopores & 99 & 1650 & 13 & 96 \\
\hline Acartia sp. nauplii & 435 & 7250 & 14 & 103 \\
\hline Eurytemora herdmani nauplii & 47 & 783 & 1 & 7 \\
\hline Centropages hamatus nauplii & 31 & 516 & - & - \\
\hline Microsetella norvegica nauplii & 12 & 200 & - & - \\
\hline M. norvegica adults and copepodites & 56 & 933 & 1 & 7 \\
\hline Oithona similis nauplii & 4 & 67 & - & - \\
\hline Pseudocalanus minutus nauplii & 9 & 150 & 2 & 14 \\
\hline Temora longicornis nauplii & 65 & 1083 & - & - \\
\hline Unid. harpacticoid nauplii & 2 & 34 & - & - \\
\hline Unid. copepod nauplii & 8 & 133 & - & - \\
\hline Unid. copepod eggs & 22 & 366 & - & - \\
\hline Hansen's nauplij & - & - & 3 & 22 \\
\hline Unid. invertebrate eggs & 10 & 167 & - & - \\
\hline \multicolumn{5}{|l|}{ Larger organisms } \\
\hline Unid. polychaete setegers & 1 & 17 & 4 & 29 \\
\hline Unid. nematodes & 2 & 34 & 1 & 7 \\
\hline Littorina sp. eggs & 1 & 17 & 6 & 44 \\
\hline Acartia longiremis adults & 12 & 200 & 32 & 235 \\
\hline Acartia sp. copepodites & 58 & 966 & 102 & 750 \\
\hline E. herdmani adults & 1 & 17 & 3 & 22 \\
\hline E. herdmani copepodites & 13 & 216 & 37 & 272 \\
\hline O. similis adults and copepodites & 3 & 50 & 15 & 110 \\
\hline P. minutus copepodites & 8 & 133 & 1 & 7 \\
\hline T. longicornis adults & 4 & 67 & 8 & 59 \\
\hline T. longicornis copepodites & 24 & 400 & 36 & 264 \\
\hline Unid. Harpacticoid adults and copepodites & 2 & 34 & 3 & 22 \\
\hline Cirripede nauplii & 15 & 250 & 38 & 279 \\
\hline Cirripede cyprids & 14 & 233 & 38 & 279 \\
\hline Volume filtered & \multicolumn{2}{|c|}{$119.8 \mathrm{~m}^{3}$} & \multicolumn{2}{|c|}{$135.9 \mathrm{~m}^{3}$} \\
\hline Settled volume & \multicolumn{2}{|c|}{$113 \mathrm{~cm}$} & \multicolumn{2}{|c|}{$82 \mathrm{~cm}$} \\
\hline
\end{tabular}


Table 6. Abundances and species composition of fish larvae (expressed as number of larvae per $100 \mathrm{~m}^{3}$ ), Damariscotta River estuary, 1979

\begin{tabular}{|c|c|c|c|c|c|c|c|c|c|c|c|c|c|c|c|c|c|c|c|c|c|c|c|}
\hline $\begin{array}{l}\text { Sample } \\
\text { date }\end{array}$ & 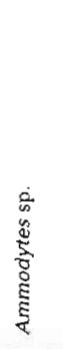 & 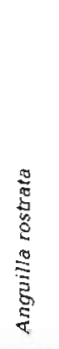 & 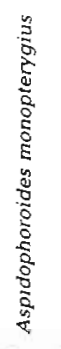 & 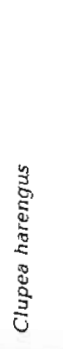 & 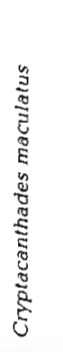 & 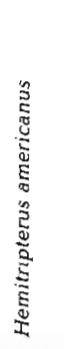 & 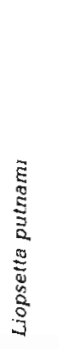 & 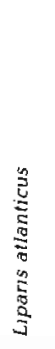 & 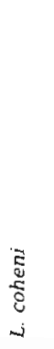 & 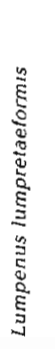 & 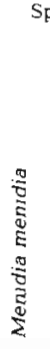 & 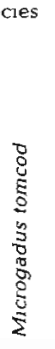 & 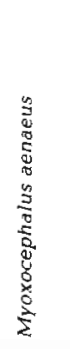 & 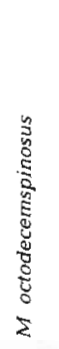 & 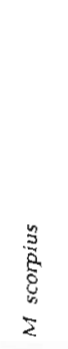 & 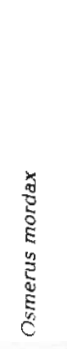 & 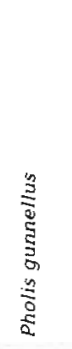 & 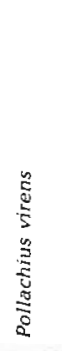 & 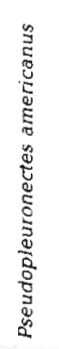 & 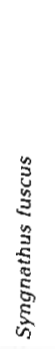 & $\begin{array}{l}5 \\
5 \\
5 \\
5 \\
5 \\
6 \\
0 \\
0 \\
5 \\
E\end{array}$ & 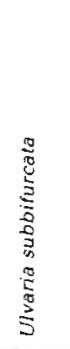 & Total \\
\hline I Feb & & & & 0.17 & & & & & 0.43 & 1.45 & & & & 0.26 & & & 1.12 & 0.09 & & & & & 3.52 \\
\hline 7 Feb & & & & 0.10 & & & & & & 0.82 & & & 0.27 & 0.92 & 0.19 & & 0.91 & & & & & & 3.21 \\
\hline $15 \mathrm{Feb}$ & & & & 0.17 & & & & & 0.09 & 0.81 & & & 0.47 & 0.62 & 0.90 & & 3.88 & & & & & & 6.93 \\
\hline $23 \mathrm{Feb}$ & & & & & 0.09 & & & & & 0.05 & & & 0.28 & 0.69 & 2.22 & & 3.35 & 0.09 & & & & & 6.76 \\
\hline $2 \mathrm{Mar}$ & & & & 0.47 & 0.18 & & & & 0.10 & 2.94 & & & 1.33 & 3.70 & 12.43 & & 17.65 & 0.18 & & & 0.10 & & 39.07 \\
\hline $8 \mathrm{Mar}$ & 0.16 & & & 0.78 & 0.08 & & & & 0.26 & 2.30 & & 0.09 & 5.96 & 4.10 & 13.83 & & 44.37 & 0.08 & & & & & 72.01 \\
\hline $16 \mathrm{Mar}$ & 0.08 & & & 1.69 & 2.95 & 0.25 & 0.33 & & 1.92 & 3.69 & & & 6.33 & 6.07 & 25.66 & & 54.64 & 1.52 & & & 0.08 & & 105.22 \\
\hline $22 \mathrm{Mar}$ & 0.16 & & & 8.55 & 0.73 & & & & 0.30 & 2.83 & & 0.08 & 12.83 & 6.56 & 17.39 & & 46.24 & 4.04 & & & & & 100.13 \\
\hline $30 \mathrm{Mar}$ & 0.50 & & & 2.91 & 1.24 & 0.34 & 0.36 & & 0.09 & 0.78 & & 0.19 & 5.16 & 0.68 & 0.67 & & 22.32 & 3.36 & & & & & 38.59 \\
\hline $6 \mathrm{Apr}$ & 0.65 & & & 2.59 & 0.08 & 0.08 & 0.73 & & & 0.08 & & & 6.40 & 0.09 & & 0.08 & 18.38 & 0.17 & & & & & 29.34 \\
\hline $11 \mathrm{Apr}$ & 0.51 & 0.17 & & 4.28 & 0.08 & & & & & & & & 0.33 & 0.09 & 0.08 & & 5.68 & 2.19 & & & & & 13.41 \\
\hline $18 \mathrm{Apr}$ & 1.01 & 0.10 & 0.56 & 4.58 & & & & 0.10 & & 0.19 & & & 0.75 & & & & 6.00 & 3.71 & & & & & 16.70 \\
\hline $25 \mathrm{Apr}$ & 1.00 & 0.09 & 0.16 & 1.75 & & & 0.59 & & & 0.09 & & & 1.54 & & & & 14.80 & 0.34 & 1.38 & & & 0.18 & 21.92 \\
\hline 4 May & 0.07 & & & 1.73 & 0.08 & & 0.16 & & & & & & 0.37 & & & 1.40 & 2.32 & 0.07 & 1.28 & & & 20.52 & 28.00 \\
\hline 9 May & & & & 1.43 & & & & & & & & & & & & 6.77 & 1.14 & 0.07 & 0.94 & & & 7.07 & 17.42 \\
\hline $18 \mathrm{May}$ & & & & & & & & & & & & & & & & 19.25 & & & 4.62 & & & 10.11 & 33.99 \\
\hline 23 May & & & & & & & & 0.09 & & & & & & & & 21.96 & & & 2.96 & & & 0.67 & 25.69 \\
\hline $30 \mathrm{May}$ & & & & & & & & 0.17 & & & & & & & & 29.72 & & & 5.42 & & & 1.40 & 36.72 \\
\hline 6 Jun & & & & & & & & & & & 0.18 & & & & & 13.42 & 0.18 & & 1.97 & & & 0.54 & 16.28 \\
\hline $13 \mathrm{Jun}$ & & & & & & & & & & & & & & & & 1.08 & & & 0.10 & & & 0.18 & 1.35 \\
\hline $20 \mathrm{Jun}$ & & & & & & & & & & & & & & & & 0.16 & & & 0.16 & 0.16 & & & 0.48 \\
\hline
\end{tabular}

Table 7. Abundances and species composition of fish larvae (expressed as number of larvae per $100 \mathrm{~m}^{3}$ ), Sullivan Harbor, 1979

\begin{tabular}{|c|c|c|c|c|c|c|c|c|c|c|c|c|c|c|c|c|c|c|c|c|c|c|}
\hline $\begin{array}{c}\text { Sample } \\
\text { date }\end{array}$ & 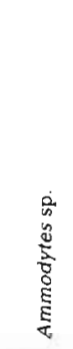 & 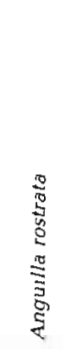 & 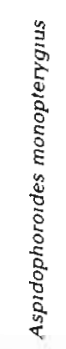 & 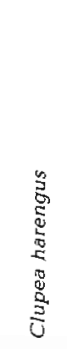 & 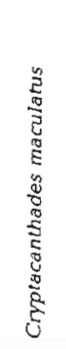 & 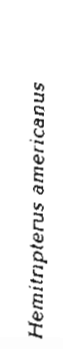 & 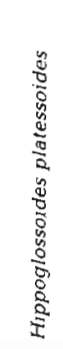 & 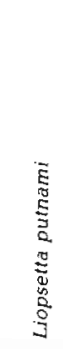 & 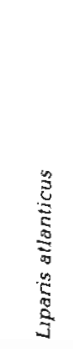 & $\begin{array}{l}\overrightarrow{8} \\
\frac{0}{8} \\
-i\end{array}$ & 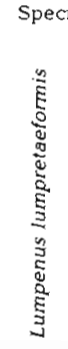 & 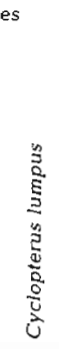 & 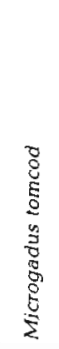 & 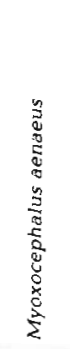 & 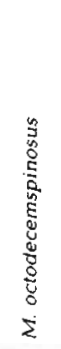 & 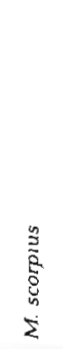 & 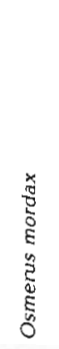 & 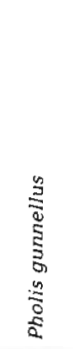 & 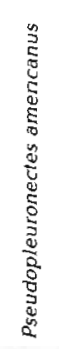 & $\begin{array}{l}5 \\
5 \\
5 \\
E \\
5 \\
0 \\
0 \\
5 \\
5\end{array}$ & 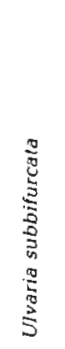 & Total \\
\hline $24 \mathrm{Jan}$ & & & & & & & & & & & 0.96 & & & & 0.14 & 0.43 & & & & & & 1.52 \\
\hline $31 \mathrm{Jan}$ & & & & & 0.09 & & & & & & 1.68 & & & & 0.26 & & & & & & & 2.03 \\
\hline $8 \mathrm{Feb}$ & & & & & 0.18 & & & & & 0.08 & 1.37 & & & & 0.50 & 0.08 & & 0.26 & & & & 2.48 \\
\hline 16 Feb & & & & & 0.19 & & & & & & 2.06 & & & & 0.75 & & & & & & & 3.00 \\
\hline 22 Feb & & & & & 0.09 & & & & & 0.10 & 4.82 & & & & 1.52 & 0.10 & & & & & & 6.62 \\
\hline 1 Mar & & & & & 0.08 & & & & & & 4.72 & & & & 0.84 & 2.31 & & 1.22 & & 0.10 & & 9.27 \\
\hline 7 Мат & 0.08 & & & & 0.33 & 0.08 & & & & 0.85 & 4.40 & & & 0.19 & 6.70 & 4.68 & & 5.36 & & & & 22.68 \\
\hline $15 \mathrm{Mar}$ & 46 & & & 0.19 & 0.94 & & & & & 0.44 & 12.05 & & & 026 & 9.78 & 5.13 & & 24.10 & & & & 53.35 \\
\hline $23 \mathrm{Mar}$ & 35.38 & & & & 2.79 & 0.70 & & & & 1.61 & 1.03 & & 0.61 & 9.83 & 4.02 & 9.83 & & 77.49 & & 0.08 & & 143.38 \\
\hline $29 \mathrm{Mar}$ & 1.13 & & & 0.49 & 4.05 & 0.33 & & & & 0.32 & 3.12 & & & 1.15 & 0.65 & & & 22.79 & & & & 34.68 \\
\hline 5 Apr & 19.02 & & & & 1.03 & 0.18 & & 0.18 & & 1.95 & 2.08 & & & 2366 & 0.89 & 0.87 & & 45.81 & & & & 95.66 \\
\hline $10 \mathrm{APr}$ & 1908 & & & 0.63 & 057 & 0.43 & & 0.09 & 0.34 & 1.47 & 2.05 & & 009 & 20.64 & 0.76 & 1.51 & & 4873 & & 0.09 & & 96.45 \\
\hline 19 Apr & 19.60 & & 0.09 & 1.83 & & & & & 1.94 & 0.71 & 2.63 & & & 071 & 0.31 & 3.96 & & 77.46 & & & & 109.23 \\
\hline $26 \mathrm{Apr}$ & 13.04 & & & 0.84 & & 0.56 & & 019 & 3.73 & 0.18 & & & & 704 & 0.09 & 0.54 & & 9.47 & & & 0.10 & 35.77 \\
\hline 3 May & 9.98 & & & 1.62 & & 0.19 & & & 19.46 & & 0.09 & & & 0.97 & & 010 & & 1.53 & 0.30 & & 0.09 & 34.34 \\
\hline $10 \mathrm{May}$ & 2.19 & 0.08 & & 2.24 & & 0.5 .3 & & & 35.13 & & & & & 0.55 & & & 115 & 1.10 & 0.87 & & & 43.83 \\
\hline $17 \mathrm{May}$ & 2.74 & & 0.09 & & & & & & 24.45 & & & & & 0.11 & & & 0.27 & 0.17 & 2.51 & & 0.36 & 27.69 \\
\hline 24 May & & & & & & 0.09 & 0.10 & & 7.36 & & & 0.09 & & & & & 048 & & 1.69 & & 0.47 & 10.29 \\
\hline $31 \mathrm{May}$ & 0.08 & & & & & & 0.09 & & 8.35 & & & & & & & & 0.18 & & 1.43 & & 1.07 & 11.20 \\
\hline 7 Jun & & & & & & & & & 19.06 & & & 0.09 & & & & & 0.09 & & 2.12 & & 0.98 & 22.35 \\
\hline 14 Jun & & & & & & & & & 9.35 & & & & & & & & 0.17 & & 7.80 & & 1.71 & 19.23 \\
\hline 21 Jun & & & & & & & & & 0.77 & & & & & & & & & & 0.69 & & 0.78 & 2.23 \\
\hline $28 \mathrm{Jun}$ & & & & & & & & & 1.08 & & & & & & & & & & 1.45 & & 3.64 & 6.17 \\
\hline $5 \mathrm{Jul}$ & & & & & & & & & 0.20 & & & 0.09 & & & & & & & 1.36 & & 0.20 & 1.85 \\
\hline
\end{tabular}


compare my results. The overall abundances of the dominant late winter species increased earlier in the Damariscotta than in Sullivan Harbor by 1 to 3 weeks. The peak abundances of these species, like the zooplankton, occurred earlier in 1980 than 1979 in each area.

\section{DISCUSSION}

The timing of the late winter-early spring phytoplankton bloom in 1979 in the Damariscotta estuary was similar to that reported by Cura (1981) for 1978 . He reported that the chlorophyll levels were generally low

Table 8. Abundances and species composition of fish larvae (expressed as number of larvae per $100 \mathrm{~m}^{3}$ ), Damariscotta River estuary, 1980

\begin{tabular}{|c|c|c|c|c|c|c|c|c|c|c|c|c|c|c|c|c|c|c|}
\hline $\begin{array}{l}\text { Sample } \\
\text { date }\end{array}$ & $\begin{array}{l}\dot{0} \\
0 \\
0 \\
8 \\
8 \\
5 \\
8 \\
0 \\
5 \\
\xi \\
\xi \\
8\end{array}$ & $\begin{array}{l}0 \\
5 \\
5 \\
5 \\
0 \\
0 \\
5 \\
5 \\
5 \\
5 \\
5\end{array}$ & 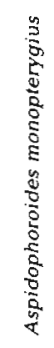 & $\begin{array}{l}3 \\
0 \\
0 \\
0 \\
0 \\
0 \\
0 \\
0 \\
0 \\
0 \\
0\end{array}$ & 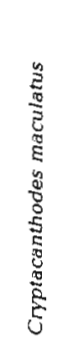 & 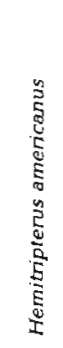 & 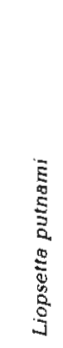 & $\begin{array}{l}0 \\
0 \\
8 \\
8 \\
8 \\
5 \\
5 \\
0 \\
0 \\
3 \\
3\end{array}$ & 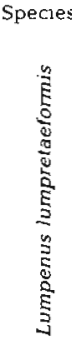 & 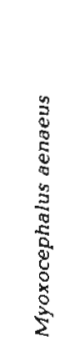 & 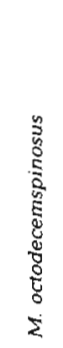 & 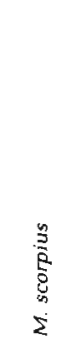 & 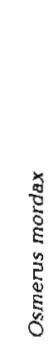 & 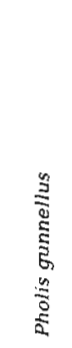 & 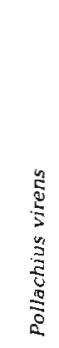 & 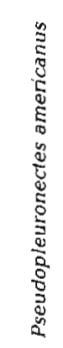 & 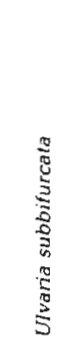 & Total \\
\hline $29 \mathrm{Jan}$ & & & & 0.18 & & & & 0.18 & 0.88 & 2.36 & 1.57 & & & 3.95 & & & & 9.12 \\
\hline $4 \mathrm{Feb}$ & & & & 0.18 & & & & & 0.53 & 1.24 & 1.41 & & & 6.50 & & & & 6.21 \\
\hline $12 \mathrm{Feb}$ & & & & 0.11 & & 0.19 & & 0.09 & 0.88 & 2.51 & 3.07 & 1.31 & & 12.03 & & & & 21.16 \\
\hline $19 \mathrm{Feb}$ & & & & 0.29 & 0.86 & & & & 1.39 & 3.10 & 2.66 & 2.79 & 0.10 & 20.66 & 0.08 & & & 31.92 \\
\hline $26 \mathrm{Feb}$ & & & & 0.33 & 0.99 & 0.16 & & 0.50 & 1.81 & 11.14 & 4.40 & 12.46 & & 42.44 & & & & 74.22 \\
\hline $4 \mathrm{Mar}$ & & & & 0.17 & 0.26 & & & 0.18 & 0.43 & 6.35 & 0.69 & 16.87 & & 37.57 & 0.08 & & & 62.51 \\
\hline $11 \mathrm{Mar}$ & & 0.19 & & 0.19 & 1.03 & & & & & 8.65 & 2.57 & 14.96 & & 29.78 & & & & 57.37 \\
\hline $25 \mathrm{Mar}$ & 1.06 & 0.35 & & 16.25 & & & & 0.87 & 0.17 & 19.00 & 2.28 & 13.91 & & 34.88 & 1.75 & & & 90.52 \\
\hline $4 \mathrm{Apr}$ & 1.16 & & & 34.22 & & 0.59 & & & 0.14 & 1.44 & 0.14 & 1.31 & & 49.31 & 0.29 & & & 88.60 \\
\hline $11 \mathrm{Apr}$ & 0.33 & 0.17 & & 6.31 & & 0.18 & & & & 0.74 & & 0.17 & & 12.13 & 1.01 & & & 21.04 \\
\hline $14 \mathrm{Apr}$ & & & & 12.51 & & 0.16 & 0.32 & & $0.0 \mathrm{~B}$ & 0.74 & & & & 3.31 & 0.08 & & & 17.21 \\
\hline $18 \mathrm{Apr}$ & 0.26 & & 0.13 & 8.73 & & & 0.09 & & & 0.94 & 0.08 & & & 7.77 & 0.08 & & & 18.13 \\
\hline 25 Арг & 0.08 & & 0.09 & 2.28 & & & 0.09 & & & 0.18 & & & & 1.02 & & & 0.16 & 3.89 \\
\hline 6 May & & & & 0.17 & & & & & & & & & 1.20 & & & 2.58 & 5.51 & 9.47 \\
\hline
\end{tabular}

Table 9. Abundances and species composition of fish larvae (expressed as number of larvae per $100 \mathrm{~m}^{3}$ ), Sullivan Harbor, 1980

\begin{tabular}{|c|c|c|c|c|c|c|c|c|c|c|c|c|c|c|c|c|c|c|c|}
\hline $\begin{array}{c}\text { Sample } \\
\text { date }\end{array}$ & 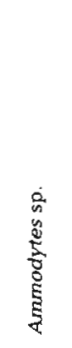 & 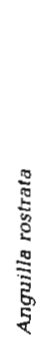 & 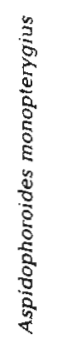 & 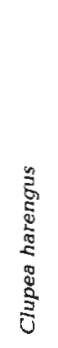 & 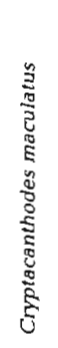 & 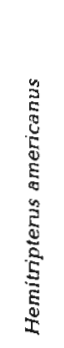 & 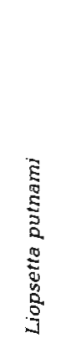 & 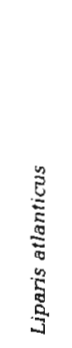 & $\begin{array}{l}\mathrm{g} \\
\mathrm{g} \\
\mathrm{E}\end{array}$ & 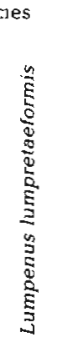 & 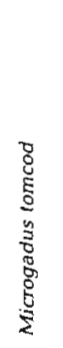 & 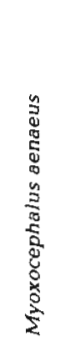 & 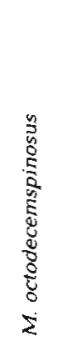 & 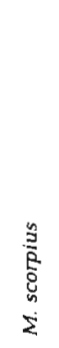 & 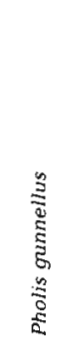 & 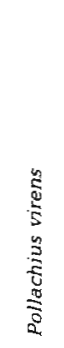 & 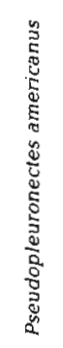 & 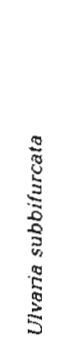 & Total \\
\hline $28 \mathrm{Jan}$ & & & & & & & & & 0.09 & 2.93 & & 0.09 & 0.56 & & 2.66 & & & & 6.33 \\
\hline 5 Feb & & & & & 0.18 & & & & & 0.63 & & & 0.35 & 0.09 & & & & & 1.25 \\
\hline $11 \mathrm{Feb}$ & & & & & 0.10 & & & & 0.10 & 0.48 & & 0.10 & 0.10 & & 1.19 & & & & 2.06 \\
\hline $18 \mathrm{Feb}$ & & & & & 0.17 & & & & 0.09 & 0.62 & & & 1.32 & & 0.27 & & & & 2.47 \\
\hline $25 \mathrm{Feb}$ & & & & & 0.94 & & & & 0.08 & 1.29 & & 0.10 & 4.46 & 0.53 & 15.70 & & & & 23.09 \\
\hline $3 \mathrm{Mar}$ & & & & & 0.41 & & & & 0.24 & 0.35 & & 0.26 & 1.89 & 1.05 & 4.45 & & & & 8.74 \\
\hline $10 \mathrm{Mar}$ & 0.09 & & & & 1.50 & & & & 0.88 & 0.52 & 0.09 & 0.97 & 6.48 & 5.81 & 20.51 & & & & 36.84 \\
\hline $26 \mathrm{Mar}$ & 10.66 & & & & 0.55 & 1.06 & & & 1.10 & & & 1.81 & 4.05 & 1.96 & 91.90 & & & & 113.09 \\
\hline $3 \mathrm{Apr}$ & 3.85 & 0.09 & & 0.19 & 1.76 & 0.46 & & & 1.40 & 1.09 & 0.09 & 1.55 & 3.54 & 2.75 & 67.71 & & & & 84.48 \\
\hline $10 \mathrm{Apr}$ & 12.07 & & & 0.33 & 0.23 & 0.84 & 0.61 & 0.17 & 0.57 & 0.09 & 0.33 & 10.71 & 0.25 & 2.44 & 41.16 & 0.09 & & & 70.05 \\
\hline $17 \mathrm{Apr}$ & 3.35 & & & 0.95 & & & & & 0.24 & & & 0.71 & & 0.08 & 6.68 & & & & 12.02 \\
\hline $24 \mathrm{Apr}$ & 11.23 & & 0.27 & 1.40 & & 0.10 & 0.10 & 1.31 & 0.38 & & & 2.49 & 0.10 & & 3.82 & & & & 21.21 \\
\hline 5 May & 11.29 & & 0.09 & 0.08 & & 0.08 & 0.15 & 1.99 & & & & & & & 0.83 & 0.15 & 0.30 & & 14.96 \\
\hline 11 May & 6.79 & & & & & & & 15.12 & & & & 0.62 & & & 0.62 & & 1.54 & 0.31 & 24.99 \\
\hline
\end{tabular}


in February $\left(0.7\right.$ to $\left.1.0 \mu \mathrm{gl}^{-1}\right)$ and reached a peak on 20 March $\left(3.6 \mu \mathrm{gl}^{-1}\right)$. He noted that the bloom that year occurred within $7 \mathrm{~d}$ after the average in situ light intensity exceeded 40 ly $\mathrm{d}^{-1}$, and that it was not triggered by a sudden influx of nutrients. Hitchcock and Smayda (1977) reported a similar response in Narragansett Bay to this apparently critical light intensity This phenomenon may explain the difference in timing of the early phytoplankton blooms between areas in my study. The late February-early March bloom in the Damariscotta in 1979 occurred before any marked influx of freshwater or increase in vertical stability and began as the water temperature was climbing above about $1^{\circ} \mathrm{C}$. In Sullivan Harbor, the late March-early April bloom also occurred as the water temperature increased above $1^{\circ} \mathrm{C}$ and did not correspond to any marked increase in vertical stability. It is quite possible that if the 1 to $1.5 \mathrm{C}^{\circ}$ change in temperature were of only minor importance, that differences in in situ light intensities may have controlled the timing of these blooms. Although not measured in this study, extinction coefficients might have been greater in Sullivan Harbor where, in addition to being shallower than the Damariscotta, the mean tidal range is about $0.5 \mathrm{~m}$ greater in Sullivan $(3.2 \mathrm{~m}$ vs $2.8 \mathrm{~m})$. It could be argued that tidal mixing resulted in a higher suspended particulate load in Sullivan Harbor, and that a greater solar elevation later in the spring was required to give a critical in situ light intensity for a phytoplankton bloom. Bigelow et al. (1940) also reported that the peak in phytoplankton in the coastal waters near $M t$. Desert Island', which is at the mouth of Frenchman Bay and Sullivan Harbor, generally lags behind the western Gulf of Maine.

The times of peak abundances of the major groups of zooplankton common to both sample areas, i.e. postnaupliar copepods, copepod nauplii and cirripede nauplii, are shown in Fig. 3. It appears that the abundance cycles of these groups in 1979 in the Damariscotta were coupled to that of the phytoplankton. Each group began to increase in numbers in late February, commensurate with rising chlorophyll concentrations. The copepod nauplii and cirripede nauplii, as well as Synchaeta sp. and Tintinnopsis sp. (Table 1), reached maximal abundances at the end of the phytoplankton bloom while the post-naupliar copepods peaked in early March. The abundance curve of cirripede nauplii in 1980 in the Damariscotta was similar in shape to the previous year but occurred earlier in the season, while the abundances of post-naupliar copepods were lower than in the previous year and fluctuated before rising abruptly on the last sample date. Lee (1975) reported that the copepods in the Damariscotta estuary had 2 abundance peaks in 1972, one in early June and the other in August and September, which more closely resembles my results in 1980 than in 1979 . The postnaupliar copepod maximum occurred much earlier in 1979 than in 1980 in the Damariscotta, suggesting that year to year variability is quite significant. The abundances of the major groups of zooplankton in Sullivan Harbor in 1979 were relatively high before the phytoplankton bloom there, and were much lower than in the Damariscotta. It is quite possible, however, that grazing pressure early in the season in Sullivan delayed the time of and suppressed the phytoplankton bloom.

The post-naupliar copepods in Sullivan Harbor in 1979 were dominated by a single species, Microsetella norvegica, which was most abundant on the first sample date (Table 2). The abundance of this species reached its lowest value on 3 May, but began to increase again until the last sample date on 28 June. The numbers of $M$. norvegica nauplii began to increase in late April. These results are consistent with those of Fish and Johnson (1937) who reported that this species 'swarmed' in Frenchman Bay in July and August of 1930 and outnumbered all other zooplankton species combined. Fish (1955) noted that spawning of $M$. norvegica in the inner Gulf of Maine began during April in 1932, and that propagation reached its peak in July and early August. He reported that there was a progressive delay in spawning to the eastward and that by September propagation had ceased in the entire Gulf in both 1931 and 1932. It appears from my results that spawning of $M$. norvegica can occur later than September or that the development of the young during summer and fall is sufficiently slow to produce a large population of adults and copepodites in winter. The presence of eggs throughout the 1979 sampling period in my study would suggest that spawning can occur during much of the year. The abundance of this species in 1980 could not be adequately assessed because of the larger mesh nets used that year.

As in Damariscotta estuary, cirripede nauplii in Sullivan Harbor appeared earlier in 1980 than in 1979. The late season increase in post-naupliar copepods also occurred earlier in 1980 . Although it is commonly accepted that the seasonal cycles of zooplankton and phytoplankton are strongly linked (Cushing, 1959), it has been demonstrated only rarely (i.e. Toner, 1981). The abundances of zooplankton in my study appeared to be strongly linked to the phytoplankton in 1979 in the Damariscotta estuary but less so in Sullivan Harbor. Rather, the zooplankton preceded the phytoplankton peak in Sullivan that year. In addition, the timing of peak abundances of zooplankton in each area in 1980 was earlier than the previous year. This was most obvious for the abundant cirripede nauplii. The release of these barnacle nauplii in the spring is usually synchronized with the phytoplankton bloom (Barnes, 
1962), and indeed this appeared to be the case in the Damariscotta in 1979, but they preceded the bloom in Sullivan Harbor. Thus, the earlier appearance of these nauplii in 1980 does not necessarily indicate that the phytoplankton bloom that year was also earlier.

The times of peak abundances of the dominant larval fish species appeared to be coupled to the spring plankton blooms. In 1979 the rises in abundance of the dominant larval fish species occurred 1 to $3 \mathrm{wk}$ earlier in the Damariscotta estuary than in Sullivan Harbor, as did the zooplankton. Also, like the zooplankton, the rises in abundance of the fish larvae occurred earlier in each sample area in 1980 than the previous year. A causative link between the zooplankton abundances and larval fish abundances seems likely. This was examined closely for Pholis gunnellus larvae (Townsend, 1983) whose survival and growth appeared to depend upon the dynamics of its planktonic food. The relation in time between the abundances of the dominant fish larvae and the plankton biomass is shown in Fig. 4 for 1979 (1980 sampling
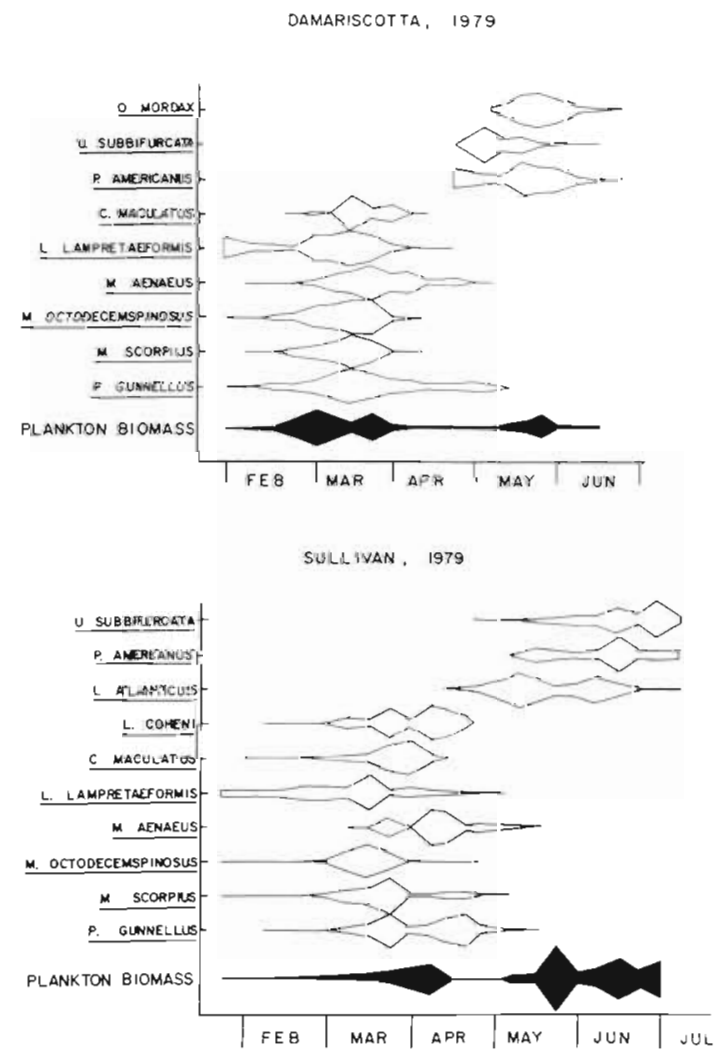

Fig. 4. Graphical representation of relative abundances of the dominant fish larvae in relation to plankton biomass for Damariscotta estuary and Sullivan Harbor, 1979. The widths of each species abundance plot is relative to itself, i.e. the widest portion indicates the time that species reached maximum abundance (Tables 1 to 4 ). Plankton biomass equals the settled volume biomass estimates from $80 \mu \mathrm{m}$ mesh $20 \mathrm{~cm}$ bongo samples ended early and these results were not plotted). These data show that the late-winter early-summer larval fish assemblages occurred in 2 groups in both Damariscotta estuary and Sullivan Harbor, and each group corresponded to distinct pulses in plankton biomass.

Acknowledgements. This work was supported in part by an NSF Research Initiation and Support Grant to the Migratory Research Institute of the University of Maine at Orono, and a grant from the National Marine Fisheries Service. Much of this work was conducted while at the Department of Oceanography, University of Maine, Ira C. Darling Center, Walpole, Maine. I thank M. Dunn, D. Hodges, R. Schnell and L. Palmer for assistance in the lab and in the field.

I thank Drs. B. J. McAlice, J. J. Graham and H. H. DeWitt for many hours of thoughtful discussion. Also, thanks to Peg Colby and Pat Oathout for typing the manuscript and Jim Rollins for drafting the figures.

\section{LITERATURE CITED}

Barnes, H. (1962). Note on variations in the release of nauplii of Balanus balanoides with special reference to the spring diatom outburst. Crustaceana 4: 118-122

Bigelow, H. B. (1926). Plankton of the offshore waters of the Gulf of Maine. Bull. U. S. Bur Fish. 40: 1-509

Bigelow, H. B. (1927). Physical oceanography of the Gulf of Maine. Bull. U. S. Bur. Fish. 40: 511-1027

Bigelow, H. B., Lillick, L. C., Sears, M. (1940). Phytoplankton and planktonic protozoa of the offshore waters of the Gulf of Maine. Part I. Numerical distribution. Trans. Am. Philos. Soc. 21: 149-191

Chenoweth, S. B. (1973). Fish larvae of the estuaries and coast of central Maine. Fish. Bull. U. S. 71: 105-113

Clarke, G. L. (1933). Diurnal migration of plankton in the Gulf of Maine and its correlation with changes in submarine irradiation. Biol. Bull. mar. biol. Lab., Woods Hole 65: $402-436$

Clarke, G. L. (1934). Further observations on the diurnal migration of copepods in the Gulf of Maine. Biol. Bull. mar biol. Lab., Woods Hole 67: 432-455

Colton, J. B., Jr., Temple, R. F., Honey, K. A. (1962). The occurrence of oceanic copepods in the Gulf of MaineGeorges Bank area. Ecology 43: 166-171

Cura, J. (1981). Physical and biological factors affecting phytoplankton growth and seasonal succession in the Damariscotta River estuary. Ph. D. thesis, University of Maine

Cushing, D. H. (1959). The seasonal variation in oceanic production as a problem in population dynamics. J. Cons. 23: $178-188$

Fish, C. J. (1955). Observations on the biology of Microsetella norvegica. Pap. mar. Biol. Oceanogr., Deep Sea Res. 3 (Suppl.): 242-249

Fish, C. J., Johnson, M. W. (1937). The biology of the zooplankton population in the Bay of Fundy and Gulf of Maine, with special reference to production and distribution. J. Biol. Bd Can. 3: 189-322

Graham, J. J., Boyar, H. C. (1965). Ecology of herring larvae in the coastal waters of Maine. ICNAF Spec. Publ. 6: 625-634

Hauser, J. W. (1973). Larval fish ecology of the Sheepscot River-Montsweag Bay estuary, Maine. Ph. D. thesis, University of Maine 
Hitchcock, G. L., Smayda, T. H. (1977). The importance of light in the initiation of the 1972-1973 winter/spring diatom bloom in Narragansett Bay. Limnol. Oceanogr. 22: 126-131

Laroche, J. L. (1980). Larval and juvenile abundance, distribution, and larval food habits of the larvae of five species of sculpins (Family: Cottidae) in the Damariscotta River estuary, Maine. Ph. D. thesis, University of Maine

Laroche, J. L. (1982). Trophic patterns among larvae of five species of sculpins (Family: Cottidae) in a Maine estuary. Fish. Bull. U. S. 80: $827-840$

Lee, W. Y (1975). Succession and some aspects of population dynamics of copepods in the Damariscotta River estuary, Maine. $\mathrm{Ph}_{1}$. D. thesis, University of Maine

Lee, W. Y., McAlice, B. J. (1979a). Seasonal succession and breeding cycles of three species of Acartia (Copepoida: Calanoida) in a Maine estuary. Estuaries 2: 228-235

Lee, W. Y., McAlice, B. J. (1979b). Sampling variability of marine zooplankton in a tidal estuary. Estuar. coast. mar. Sci. 8: 565-582

Legaré, J. E. H., MacLellan, D. C. (1960). A qualitative and quantitative study of the plankton of the Quoddy region in 1957 and 1958 with special reference to the food of the herring. J. Fish. Res. Bd Can. 17: 409-448

Lillick, L. C. (1940). Phytoplankton and planktonic protozoa of the offshore waters of the Gulf of Maine. Part II. Qualitative composition of the planktonic flora. Trans. Am. Philos. Soc. 31: 193-237

Lorenzen, C. J. (1966). A method for the continuous measurement of in-vivo chlorophyll concentration. Deep Sea Res. 13: $223-227$

McAlice, B. J. (1973). Plankton. In: Survey of the hydrography, sediments, plankton, benthos and the commercially important plants and animals including finfish, in the Montsweag Bay-Back River area. Preoperational Summary. Maine Yankee Atomic Power Co., Augusta, Maine, p. $31-78$

Platt, T., Dickie, L. M., Trites, R. W. (1970). Statistical heterogeneity of phytoplankton in a near-shore environment. J. Fish. Res. Bd Can. 32: 347-366

Posgay, J. A., Marak, R. R. (1981). The MARMAP bongo zooplankton samplers. J. Northw. Atl. Fish. Sci. 1: 91-99

Redfield, A. C. (1939). The history of a population of Limacina retroversa during its drift across the Gulf of Maine. Biol. Bull mar. biol. Lab., Woods Hole 76: 26-47

Redfield, A. C. (1941). The effect of the circulation of water on the distribution of the Calanoid community in the Gulf of Maine. Biol. Bull. mar. biol. Lab., Woods Hole 80: 86-110
Redfield, A. C., Beale, A. (1940). Factors determining the distribution of populations of chaetognaths in the Gulf of Maine. Biol. Bull. mar. biol. Lab., Woods Hole 79: 459-484

Shaw, R. F. (1981). Seasonal species composition, diversity, spatial distributions, and tidal retention and transport of ichthyoplankton in the Sheepscot River-Back River-Montsweag Bay estuarine system, Maine. Ph. D. thesis, University of Maine

Sherman, K. (1965). Seasonal and areal distribution of Gulf of Maine coastal zooplankton, 1963. ICNAF Spec. Publ. 6: $611-623$

Sherman, K. (1966). Seasonal and areal distribution of zooplankton in the coastal waters of the Gulf of Maine, 1964. U. S. Fish Wildl. Serv., Spec. Sci. Rep. Fish. 530: 1-11

Sherman, K. (1968). Seasonal and areal distribution of zooplankton in the coastal waters of the Gulf of Maine, 1965 and 1966. U. S. Fish Wildl. Serv., Spec. Sci. Rep. Fish. 562: $1-11$

Sherman, K. (1970). Seasonal and areal distribution of zooplankton in coastal waters of the Gulf of Maine, 1967 and 1968. U. S. Fish Wildl. Serv., Spec. Sci. Rep. Fish. 594: 1-8

Sherman, K., Perkins, H. C. (1971). Seasonal variations in the food of juvenile herring in coastal waters of Maine. Trans. Am. Fish. Soc. 100: 121-124

Toner, R. C. (1981). Interrelationships between biological, chemical and physical variables in Mount Hope Bay, Massachusetts. Estuar. coast. Shelf Sci. 12: 701-712

Townsend, D. W (1981). Comparative ecology and population dynamics of larval fishes and zooplankton in two hydrographically different areas on the Maine coast. $\mathrm{Ph}$. D. thesis, University of Maine

Townsend, D. W. (1983). The relations between larval fishes and zooplankton in two inshore areas of the Gulf of Maine. J. Plankton Res. 5 (2): 145-173

Townsend, D. W., Graham, J. J (1981). Growth and age structure of larval Atlantic herring. Clupea harengus harengus, in the Sheepscot River estuary, Maine, as determined by daily growth increments in otoliths. Fish. Bull. U. S. $79: 123-130$

Willey, A. (1913). Notes on plankton collected across the mouth of the St. Croix River opposite to the Biological Station at St. Andrews, New Brunswick, in July and August, 1912. Proc. Zool. Soc. Lond. 1913: 283-292

Willey, A. (1915). The plankton in St. Andrews Bay. Contr. Can. Biol. for 1911-1914:1-9

Yentsch, C. S., Menzel, D. W. (1963). A method for the determination of phytoplankton chlorophyll and paeophytin by fluorescence. Deep Sea Res. 10: 221-231 